\title{
Bedaquiline exposure in pregnancy and breastfeeding in women with rifampicin-resistant tuberculosis
}

\author{
Richard Court ${ }^{1}$, Kamunkhwala Gausi ${ }^{1}$, Buyisile Mkhize ${ }^{1}$, Lubbe Wiesner ${ }^{1}$, Catriona \\ Waitt $^{2}$, Helen McIlleron ${ }^{1}$, Gary Maartens ${ }^{1}$, Paolo Denti ${ }^{1}$, and Marian Loveday ${ }^{3}$ \\ ${ }^{1}$ University of Cape Town \\ ${ }^{2}$ University of Liverpool Institute of Translational Medicine \\ ${ }^{3}$ Medical Research Council of South Africa
}

November 18, 2021

\begin{abstract}
Aim We aimed to explore the effect of pregnancy on bedaquiline pharmacokinetics and describe bedaquiline exposure in the human milk of mothers treated for rifampicin-resistant TB, where there is no human data available. Methods We performed a longitudinal pharmacokinetic study in pregnant women treated for rifampicin-resistant TB to explore the effect of pregnancy on bedaquiline exposure. Pharmacokinetic sampling was performed at four time-points over six hours in the third trimester, and again at approximately six weeks postpartum. We obtained serial human milk samples from breastfeeding mothers, and a single plasma sample taken from breastfed and non-breastfed infants to assess bedaquiline exposure. We used liquid chromatography-tandem mass spectrometry to perform the human milk and plasma bedaquiline assays, and population pharmacokinetic modelling to interpret the bedaquiline concentrations. Results We recruited 13 women, six of whom completed the ante- and post-partum PK sampling. All participants were HIV-positive on antiretroviral therapy. We observed lower ante- and post-partum bedaquiline exposures than reported in non-pregnant controls. Bedaquiline concentrations in human milk were higher than maternal plasma (milk to maternal plasma ratio: 24:1). A single random plasma bedaquiline and M2 concentration was available in four infants (median age: 6.5 weeks): concentrations in the one breastfed infant were similar to maternal plasma concentrations; concentrations in the three non-breastfed infants were detectable but lower than maternal plasma concentrations. Conclusion We report low exposure of bedaquiline in pregnant women treated for rifampicin-resistant TB. Bedaquiline significantly accumulates in human milk; breastfed infants receive $\mathrm{mg} / \mathrm{kg}$ doses of bedaquiline equivalent to maternal doses.
\end{abstract}

\section{Introduction}

Acquisition of data quantifying the exposure of second-line TB drugs in pregnant woman treated for rifampicin-resistant TB (RR-TB) is a priority. Until recently, pregnant and breastfeeding women have typically been excluded from clinical trials of new drugs, including TB treatment. (1) The World Health Organization (WHO) currently recommends individualised treatment regimens with drugs with a preferred safety profile for pregnant women with RR-TB, (2) although there are limited human data guiding these recommendations.

Bedaquiline is a group A drug, recommended for inclusion in all RR-TB treatment regimens, and can be used in pregnant women, although safety data is lacking. The design of pharmacokinetic studies to explore the effect of pregnancy on long half-life drugs like bedaquiline is challenging, as cumulative drug concentrations could mask pregnancy-related effects on drug exposure. Physiological changes in pregnancy result in decreased concentrations of many drugs, particularly in the third trimester. Pharmacokinetics in pregnancy may be complex: one of the reasons for reduced drug concentrations in pregnancy is the reduction in 
plasma concentrations of the two key drug-binding proteins: albumin and $\alpha 1$-acid glycoprotein. (3) Reduction in these binding protein concentrations reduces the total (bound and unbound) concentrations of drugs, but the unbound fraction typically increases, resulting in unbound drug concentrations that are similar to non-pregnant women. As only the unbound drug is pharmacologically active, recommendations to increase the dose of drugs in pregnancy to approximate total concentrations in non-pregnant women could therefore increase the risk of toxicity. However, pregnancy also increases several drug clearance mechanisms, which could reduce unbound drug concentrations. Although measurement of unbound bedaquiline concentrations is preferable to rationally optimize dosing in pregnancy (bedaquiline is $>99 \%$ protein-bound) (4), an understanding of the effect of pregnancy on total bedaquiline concentrations would provide a much-needed foundation to understand the effect of pregnancy on the unbound fraction.

Data on the secretion of key drugs for RR-TB into human milk are scarce. The studies describing RR-TB drug exposure in human milk are small with little or no infant plasma pharmacokinetic data available the study designs are also unclear or unstated. Linezolid (5), levofloxacin (6) and cycloserine (7) penetrate poorly into human milk and exposure to breastfed infants is therefore likely to be low. Clofazimine, in contrast, demonstrates effective human milk penetration with skin discoloration observed in the infants of breastfeeding mothers treated with clofazimine for leprosy; $(8,9)$ clofazimine exposure in human milk in the context of mothers treated for TB is unfortunately lacking. Animal studies have shown that bedaquiline is concentrated in rat milk with concentrations 6- to 12- fold higher than the concentration in maternal plasma (10), but there are currently no human data available. Information on clinically relevant infant exposure to RR-TB drugs through breastfeeding with mother-infant pairs has not been done, and is an important knowledge gap.

An international consensus panel on the inclusion of pregnant and postpartum women in TB drug trials, convened by the NIH, identified the safety, tolerability and pharmacokinetics of novel agents and regimens for treatment of RR-TB as research priorities (11). It is ethically imperative to study drug dosing and safety in populations where drugs are used - this has not been done satisfactorily for RR-TB. (12) We therefore conducted an observational study of bedaquiline exposure in pregnant and breastfeeding women with RR-TB.

\section{Method}

Study design

We performed a longitudinal pharmacokinetic study in pregnant women [?] 18 years of age treated for RR-TB, and their infants, at King Dinuzulu Hospital in Durban, Kwazulu-Natal - nested within a cohort, which has been previously described. (13) KDH is a specialist provincial RR-TB hospital where, until recently, all pregnant women with RR-TB were referred for care. With some individual regimen variability, all participants were treated with a minimum of five drugs including bedaquiline. Other drugs included: pyrazinamide, isoniazid, clofazimine, linezolid, moxifloxacin, and less commonly: ethambutol, terizidone, levofloxacin, ethionamide and para-aminosalycylic acid. We performed pharmacokinetic sampling pre-dose, and two, four and six hours post-dose in the third trimester of pregnancy ([?]28 weeks), and at the six week postpartum visit. Dosing on both sampling days was observed after a standard breakfast; the tablets/capsules were ingested with $250 \mathrm{~mL}$ of water. Considering bedaquiline is dosed three times a week (after the two week loading dose), it was not always logistically possible to schedule pharmacokinetic sampling on a day when bedaquiline was administered. We therefore recorded the last date and time when bedaquiline was dosed to interpret the exposures with our modelling. All concurrent medications, including antiretroviral therapy were recorded. If available, human milk samples were taken from breastfeeding mothers by manual expression at the same timepoints that blood was drawn at the postpartum visit (i.e. pre-dose, and two, four and six hours post-dose); samples were frozen within 30 minutes of sampling at minus $80^{\circ} \mathrm{C}$. To evaluate infant drug exposure, a single random plasma sample was taken from infants at the postpartum visit. If applicable, the time of the most recent breastfeeding prior to the infant blood draw was recorded.

Pharmacokinetics

Plasma and human milk samples were stored at minus $80^{\circ} \mathrm{C}$ and transported to the University of Cape 
Town, Division of Clinical Pharmacology laboratory where total plasma and human milk bedaquiline assays were performed using liquid chromatography with tandem mass spectrometry. The plasma assay for total bedaquiline has previously been described. (14) Bedaquiline and its M2 metabolite in human milk were analysed with a validated assay developed at the Division of Clinical Pharmacology laboratory; the standards and quality checks were performed using blank donated human milk. The extraction procedure consisted of protein precipitation and solid phase extraction, followed by gradient liquid chromatography on an Agilent Poroshell 120 SB-C18 $(2.1 \mathrm{~mm}$ x $50 \mathrm{~mm}, 2.7 \mu \mathrm{m})$ analytical column with tandem mass spectrometry detection. An AB Sciex API 3000 mass spectrometer at unit resolution in the multiple reaction monitoring mode was used to monitor the transitions of the protonated precursor ions $\mathrm{m} / \mathrm{z} 555.1, \mathrm{~m} / \mathrm{z} 561.1, \mathrm{~m} / \mathrm{z} 541.1$, and m/z 545.1 to the product ions $\mathrm{m} / \mathrm{z} 58.2, \mathrm{~m} / \mathrm{z} 64.1, \mathrm{~m} / \mathrm{z} 480.3$, and $\mathrm{m} / \mathrm{z} 480.4$ for bedaquiline, TMC207-d6, M2, and M2-d3C13, respectively. Electro Spray Ionisation was used for ion production. The calibration curves fitted quadratic (weighted by $1 / \mathrm{x}$ ) regressions based on peak area ratios over the ranges $0.0780-5.00 \mu \mathrm{g} / \mathrm{mL}$ for bedaquiline and $0.0312-2.00 \mu \mathrm{g} / \mathrm{mL}$ for M2. The combined accuracy (\%Nom) and precision (\%CV) statistics of the lower limit of quantification, low, medium, and high quality controls of bedaquiline and M2 during intra- and inter validations were between $96.7 \%$ and $106.5 \%$, and $3.4 \%$ and $7.5 \%$, respectively.

\section{Modelling}

Bedaquiline concentrations were interpreted using population pharmacokinetic modelling in NONMEM version 7.4.5 (15). Perl-speaks-NONMEM version 5.2.6, Pirana version 3.0, and $\mathrm{R}$ with the package xpose4 were used to facilitate the model development process, data manipulation, and generation of model diagnostics (16). As a starting point, we used a published population pharmacokinetic model of bedaquiline in non-pregnant adults with HIV and drug-resistant tuberculosis (14). Briefly, the published model consists of three disposition compartments for bedaquiline and one disposition compartment for M2. There was a correlation between bedaquiline and M2 between-subject-variability on clearance, and residual variabilities. The effect of body weight on all disposition parameters was included using allometric scaling, and albumin also affects the drug disposition parameters. The co-administration of ritonavir-boosted lopinavir reduced bedaquiline and M2 clearance by $65 \%$ and $42 \%$, respectively. Molar concentrations were used during model development to account for mass balance between bedaquiline and its metabolite M2. Participant albumin information were not captured in the current study, therefore we imputed a reported albumin concentration from a previously study in South Africa patients with RR-TB (17).

When analysing the data, we first fit the original model as published, without re-estimating any of the population parameters, but using the study covariate, doses and dosing regimen information. This is similar to using the current data as an "external" validation of the model, i.e. assessing how the previous model predicts the current data based solely on covariate information and assuming no effect of pregnancy (which was not part of the origina model). Afterwards, we attempted to use the data to re-estimate parameter values, using the general principles of model development including drops in NONMEM objective function value $(\mathrm{OFV})$ for assessment of statistical significance and inspection of diagnostic plots.

\section{Calculation of infant bedaquiline intake with human milk}

To estimate how much bedaquiline is ingested per day by a typical child breastfed by a mother receiving bedaquiline, we assumed an average infant milk ingestion of $0.15 \mathrm{~L} / \mathrm{kg} /$ day (18). The following equation was used to calculate the infant dose (19):

$$
D_{\text {infant }}=C_{m} \bullet V_{m}
$$

where $V m$ is the volume of milk ingested by breastfeeding, and $C_{m}$ is the drug concentration in human milk. This was calculated using the formula below:

$$
C_{m}=M: P \bullet C_{\mathrm{pss}}
$$


where $C_{p s s}$ is the average maternal plasma level at a steady-state, and $M: P$ is the human milk-to-plasma ratio.

Ethics

Ethics approval for the study was granted by the South African Medical Research Council Ethics Committee (EC017-6/2016), and the University of Cape Town Human Research Ethics Committee (HREC: 666/2018). Informed consent was taken from all participants in a language of their choice (either English or isiXhosa).

\section{Results}

\section{Study profile}

Bedaquiline pharmacokinetic samples were available from 13 women in the third trimester of pregnancy, at 30 (IQR: 25 - 37) weeks gestation, six of whom returned for postpartum sampling at 7 (IQR: 6.5 - 8) weeks. Seventy-one plasma samples of bedaquiline parent and metabolite concentration were available for analysis. Participant characteristics are shown in Table 1. All participants were living with HIV were treated with antiretroviral therapy (ART), most commonly on nevirapine-based ART $(n=10,83.3 \%)$, but one woman received lopinavir/ritonavir. Serial human milk samples at the same time-points that plasma was sampled, were available in two breasfeeding participants. A single random plasma bedaquiline concentration was available from four infants on the postpartum PK sampling day, of whom one was breastfed. The serial post-dose bedaquiline concentrations at each time point are shown in Table 2 .

\section{Pharmacokinetic model}

When we used the published model (14) to predict the expected exposures in these patients (thus using the original population parameter estimates and assuming no effect of pregnancy), the model overpredicted both bedaquiline and M2 concentration on both antepartum and postpartum visits, as presented in the visual predictive check (VPC) inFigure 1 . The VPC shows that the PK terminal elimination phase of the participant not on lopinavir/ritonavir were approximately $50 \%$ lower that the model prediction (for both the metabolite and parent) as illustrated by the deviation of the $50^{\text {th }}$ percentiles of the observations (red line) from the median of the model predicted confidence interval (black line). If the pharmacokinetic parameters in this study were in line with the previous report, we would have expected to observe higher bedaquiline concentrations. Only the data from the participant co-administered lopinavir/ritonavir, who had higher bedaquiline concentrations due to a drug-interaction, was in line with the model prediction.

When encountered several challenges when attempting to fit the original model to the current data by reestimating the parameter values. The model structure is complex, with multiple disposition compartments, and the current data did not reliably support the re-estimation of all parameters - some of the parameter estimates obtained when attempting to re-fit were unstable and/or implausible. In other words, while the model could be adapted to fit the study data, this could be achieved in multiple different ways, e.g. assuming a larger clearance or lower bioavailability (both pre- and postpartum), but also a larger peripheral volume of distribution. We experienced further complications when trying to estimate a significant difference between the two pharmacokinetic sampling visits, i.e. possibly due to pregnancy status. All the scenarios were nearly equivalent in terms of goodness of fit, and there was no meaningful difference in terms of statistical significance, thus leaving the choice largely in the domain of speculation. Choosing a different scenario (on which a difference is ascribed to) would imply a different interpretation of the results, and if the different options for the model were to be used to predict concentrations and suggest dose adjustments they could come to very different conclusions. We also attempted to use a frequentist prior approach (20) to try and stabilise the parameter estimates, but the results became highly dependent on the assumptions on the prior precision of each parameter, thus not solving the problem. For this reason, we decided to simply use the model as originally published and acknowledge that the concentrations we observed are lower than expected, assuming that the pharmacokinetics are the same as non-pregnant patients. 
A graphical overview of the infant and human milk data is provided in Figures 2 and 3, together with the plasma concentrations in the respective mothers. The PK profiles for bedaquiline and M2 are shown: maternal plasma concentrations ante- and postpartum, human milk and infant concentrations. To calculate the human milk to maternal plasma (M:P) ratio, we used matched milk and plasma samples captured more than 24 hours after the last bedaquiline dose. This was done because during drug absorption the ratio was rapidly varying and the later samples better reflect the steady-state equilibrium of bedaquiline. We observed an average M:P ratio of 24:1 and 5.9:1 for bedaquiline and M2, respectively. Therefore, assuming a maternal average steady-state concentration of $0.3 \mathrm{mg} / \mathrm{L}$, the infant bedaquiline dose would be $1.08 \mathrm{mg} / \mathrm{kg} / \mathrm{day}$. Similarly, assuming the M2 average steady-state concentration is 0.1, the infant M2 dose would be 0.09 $\mathrm{mg} / \mathrm{kg} /$ day. In comparison, a 70-kg individual administered the standard dose of $200 \mathrm{mg}$ bedaquiline three times a week would result in approximately $1.22 \mathrm{mg} / \mathrm{kg} /$ day dose of bedaquiline.

Bedaquiline and M2 concentrations in the infant who was breastfed were similar to maternal plasma concentrations, while for the three infants who were not breastfed, bedaquiline and M2 concentrations were detectable but lower than maternal plasma values (see Figures 2 and 3).

\section{Discussion}

To our knowledge, this report is the first description of the exposure of bedaquiline in pregnant women. We found bedaquiline and M2 exposure in pregnant women to be approximately $50 \%$ lower than expected in non-pregnant patients (14). Although we were underpowered, we found no significant difference between ante- and postpartum exposures.

There are several possible reasons for the low bedaquiline exposures we observed in the third trimester. First, increased metabolism of bedaquiline is a possible explanation - pregnancy is known to induce CYP3A4, which is the major route of bedaquiline metabolism (21). The increase in CYP3A4 expression would lead to higher clearance and lower bioavailability of bedaquiline, since it is present in both entero- and hepatocytes. Second, pregnancy reduces plasma albumin concentrations, to which bedaquiline is highly bound (22). The unbound fraction of bedaquiline may therefore increase, subsequently increasing its clearance and tissue distribution. In such a scenario, the total (bound+unbound) concentrations of bedaquiline in plasma would decrease, but this effect could be counter balanced by the large unbound fraction, thus maintaining relatively unchanged unbound levels. However, exploration of free bedaquiline exposure is required before a recommendation for a dose adjustment can be made. Finally, changes in body size (and possibly composition) may have affected bedaquiline disposition, but it is unlikely that the increased weight in pregnancy affected the exposure of bedaquiline as we used allometric scaling to account for this in the model, and changes in body size alone are therefore unlikely to explain the decreased bedaquiline concentrations we observed.

Similarly, we observed lower-than-expected bedaquiline levels at the postpartum visit. While it is generally accepted that pharmacokinetic sampling approximately six weeks postpartum is a reasonable time-point to allow the physiologic changes related to pregnancy to subside (23), there are some limitations in using this timeline as a control when exploring the effect of pregnancy on drugs with a long half-life such as bedaquiline. Given that the terminal half-life of bedaquiline is more than five months, (4) any change in pharmacokinetic parameters may only become apparent on drug exposure after a considerable amount of time, possibly months. Thus, even if most of the pregnancy effects (if any) had reversed in the first weeks after delivery, there may not have been sufficient time for the exposure of bedaquiline to reach a new equilibrium before the scheduled postpartum pharmacokinetic visit. An alternative explanation is that adherence could have decreased in the postpartum period; a systematic review reported poor postpartum adherence in patients on ART (24). Sub-therapeutic bedaquiline exposures could affect clinical outcomes and increase the risk of selecting for drug resistance.

We observed concerningly high concentrations of bedaquiline in the human milk samples we analysed, markedly higher than the maternal bedaquiline plasma concentrations, in keeping with the findings of an animal study. (10) The breastfeeding infant had a plasma bedaquiline concentration similar to maternal plasma (Figure 2), which could have implications for infant safety. In a previous animal study, rat pups who 
were breastfed to mothers treated with bedaquiline were reported to have low body weight. (10) In contrast, therapeutic concentrations of bedaquiline in infants (possible with long half-life drugs, which accumulate slowly, such as bedaquiline) could potentially be protective in infants exposed to RR-TB, obviating the need for TB preventive therapy. The three infants who were not breastfed had sub-therapeutic bedaquiline concentrations, likely from transplacental exposure, which could select for drug resistance should the infants develop RR-TB.

The main factors determining the transfer of a drug into human milk are its physicochemical characteristics (such as lipid solubility and degree of ionisation at different $\mathrm{pH}$ conditions) and its plasma pharmacokinetics (25). Fat-soluble drugs like bedaquiline cross lipid-protein cell membranes easily, hence transferring readily into human milk (25). The ease, with which drug molecules cross cellular membranes, depends on the drug's degree of ionisation, which may vary in different $\mathrm{pH}$ conditions. Weak bases like bedaquiline (pKa $=8.9)(26)$ tend to be slightly less ionised in plasma compared to milk. This means that unionised plasma bedaquiline will transfer into human milk, were it is more likely to be ionised, favouring milk accumulation of the drug (27). Transfer of drugs into human milk may also be greater in drugs with a low affinity for maternal plasma proteins, but bedaquiline is highly protein-bound (>99.9\%). (4) An additional factor is molecular weight, as drugs with low weight $(<200 \mathrm{Da})$ reach human milk more easily, but the molecular weight of bedaquiline is $555.504 \mathrm{Da}$ (28). Drugs, which have a long plasma half-life and therefore accumulate, such as bedaquiline, are prone to transfer into human milk compared with molecules which are cleared rapidly. The high concentration of bedaquiline in human milk suggests that the mammary glands could be a clearing site for bedaquiline. Excretion could be significant, since, on average, a baby consumes about 0.15 $\mathrm{L} / \mathrm{kg}$ /day of human milk (18). Moreover, bedaquiline metabolism in breast tissue cannot be excluded, as there are contradictory reports on the expression of CYP3A4 in human breast tissue. (29-31)

Our study has several limitations. First, we did not measure unbound bedaquiline concentrations or albumin levels, so we are unable to conclusively determine if the reasons for the low concentrations observed are related to protein binding. Second, there was a high rate of participant loss to follow up, which limited our sample size, as many participants were unable for logistical reasons, to complete the postpartum PK sampling day. Third, PK sampling was not always performed on a day when bedaquiline was scheduled to be administered (dosing is three times a week). Although this was accounted for in our modelling, considering we did not use an adherence measure, the date and time of the last bedaquiline dose was obtained via participant self-report, which could be unreliable.

We report low exposures of bedaquiline in this series of pregnant women treated for RR-TB. Future studies should analyse bound and unbound bedaquiline concentrations with adherence measures to better understand the effect of pregnancy on bedaquiline exposure, and assess whether a different dosing recommendation for bedaquiline in pregnancy is warranted. Bedaquiline appeared to significantly accumulate into human milk, which could be an exposure risk for breastfeeding babies and should therefore be investigated.

Acknowledgments

The authors would like to acknowledge the participants who volunteered for the study, and Sindisiwe Hlangu for her assistance with study implementation.

Conflicts of interest

The authors declare no conflicts of interest

Funding

The University of Cape Town Clinical PK Laboratory is supported in part via the Adult Clinical Trial Group (ACTG), by the National Institute of Allergy and Infectious Diseases (NIAID) of the National Institutes of Health under award numbers UM1 AI068634, UM1 AI068636, and UM1 AI106701; as well as the Infant Maternal Pediatric Adolescent AIDS Clinical Trials Group (IMPAACT), funding provided by National Institute of Allergy and Infectious Diseases (U01 AI068632), The Eunice Kennedy Shriver National Institute of Child Health and Human Development, and National Institute of Mental Health grant AI068632. The 
content is solely the responsibility of the authors and does not necessarily represent the official views of the sponsors. Catriona Waitt is supported by a Wellcome Clinical Research Career Development Fellowship $222075 / \mathrm{Z} / 20 / \mathrm{Z}$.

Data availability

The data that support the findings of this study are available from the corresponding author upon reasonable request.

\section{References}

1. Gupta A, Hughes MD, Garcia-Prats AJ, McIntire K, Hesseling AC. Inclusion of key populations in clinical trials of new antituberculosis treatments: Current barriers and recommendations for pregnant and lactating women, children, and HIV-infected persons. PLoS Med [Internet]. 2019;16(8):1-26. Available from: http://dx.doi.org/10.1371/journal.pmed.1002882

2. WHO. WHO Consolidated Guidelines on Tuberculosis, Module 4: Treatment - DrugResistant Tuberculosis Treatment [Internet]. 2021 [cited 2021 Oct 12]. Available from: https://www.who.int/publications/i/item/9789240007048

3. Schalkwijk S, Greupink R, Burger D. Free dug concentrations in pregnancy: Bound to measure unbound? Br J Clin Pharmacol. 2017;83(12):2595-8.

4. van Heeswijk RPG, Dannemann B, Hoetelmans RMW. Bedaquiline: a review of human pharmacokinetics and drug-drug interactions. J Antimicrob Chemother. 2014 Sep;69(9):2310-8.

5. Lim FH, Lovering AM, Currie A, Jenkins DR. Linezolid and lactation: measurement of drug levels in breast milk and the nursing infant. J Antimicrob Chemother. 2017;72(9):2677-8.

6. Cahill JB, Bailey EM, Chien S, Johnson GM. Levofloxacin secretion in breast milk: A case report. Pharmacotherapy. 2005;25(1):116-8.

7. Tran J, Montakantikul P. The safety of antituberculosis medications during breastfeeding. J Hum Lact. 1998;14(4):337-40.

8. Loveday M, Hlangu S, Furin J. Breastfeeding in women living with tuberculosis. Int J Tuberc Lung Dis. 2020;24(9):880-91.

9. Ozturk Z, Tatliparmak A. Leprosy treatment during pregnancy and breastfeeding: A case report and brief review of literature. Dermatol Ther. 2017;30(1):2016-7.

10. FDA. Full prescribing information: SIRTURO [Internet]. [cited 2021 Oct 12]. Available from: https://www.accessdata.fda.gov/drugsatfda_docs/label/2012/204384s000lbl.pdf

11. Gupta A, Mathad JS, Abdel-Rahman SM, Albano JD, Botgros R, Brown V, et al. Toward Earlier Inclusion of Pregnant and Postpartum Women in Tuberculosis Drug Trials: Consensus Statements from an International Expert Panel. Clin Infect Dis. 2015;62(6):761-9.

12. Gupta A, Hughes MD, Garcia-Prats AJ, McIntire K, Hesseling AC. Inclusion of key populations in clinical trials of new antituberculosis treatments: Current barriers and recommendations for pregnant and lactating women, children, and HIV-infected persons. PLoS Med. 2019;16(8):1-26.

13. Loveday M, Hughes J, Sunkari B, Master I, Hlangu S, Reddy T, et al. Maternal and Infant Outcomes among Pregnant Women Treated for Multidrug/Rifampicin-Resistant Tuberculosis in South Africa. Clin Infect Dis. 2021;72(7):1158-68.

14. Brill MJE, Svensson EM, Pandie M, Maartens G, Karlsson MO. Confirming model-predicted pharmacokinetic interactions between bedaquiline and lopinavir/ritonavir or nevirapine in patients with HIV and drug-resistant tuberculosis. Int J Antimicrob Agents. 2017;49(2):212-7. 
15. Boeckmann AJ, Beal SL, Sheiner LB. NONMEM User's Guide, Part V. Introductory Guide. NONMEM Proj Gr. 2011;(April):48.

16. Keizer RJ, Karlsson MO, Hooker A. Modeling and Simulation Workbench for NONMEM: Tutorial on Pirana, PsN, and Xpose. CPT Pharmacometrics Syst Pharmacol. 2013 Jun;2(6):e50.

17. Ngwalero P, Brust JCM, van Beek SW, Wasserman S, Maartens G, Meintjes G, et al. Relationship between Plasma and Intracellular Concentrations of Bedaquiline and Its M2 Metabolite in South African Patients with Rifampin-Resistant Tuberculosis. Antimicrob Agents Chemother [Internet]. 2021 Oct 18;65(11):e0239920. Available from: https://journals.asm.org/doi/10.1128/AAC.02399-20

18. Wilson JT. Determinants and Consequences of Drug Excretion in Breast Milk. Drug Metab Rev. 1983 Jan;14(4):619-52.

19. Koren G. Maternal-Fetal Toxicology: A Clinician's Guide. Third edit. Vol. 149, Archives of Pediatrics \& Adolescent Medicine. New York: American Medical Association; 1995. 177-221 p.

20. Chan Kwong AHXP, Calvier EAM, Fabre D, Gattacceca F, Khier S. Prior information for population pharmacokinetic and pharmacokinetic/pharmacodynamic analysis: overview and guidance with a focus on the NONMEM PRIOR subroutine. Journal of Pharmacokinetics and Pharmacodynamics. 2020. p. 1-16.

21. Svensson EM, Aweeka F, Park J-G, Marzan F, Dooley KE, Karlsson MO. Model-Based Estimates of the Effects of Efavirenz on Bedaquiline Pharmacokinetics and Suggested Dose Adjustments for Patients Coinfected with HIV and Tuberculosis. Antimicrob Agents Chemother. 2013 Jun;57(6):2780-7.

22. Feghali MN, Mattison DR. Clinical Therapeutics in Pregnancy. J Biomed Biotechnol. 2011 Jul;2011:113.

23. Eke AC, Olagunju A, Momper J, Penazzato M, Abrams EJ, Best BM, et al. Optimizing Pharmacology Studies in Pregnant and Lactating Women Using Lessons From HIV: A Consensus Statement. Clin Pharmacol Ther. 2021;110(1):36-48.

24. Nachega JB, Uthman OA, Anderson J, Peltzer K, Wampold S, Cotton MF, et al. Adherence to antiretroviral therapy during and after pregnancy in low-income, middle-income, and high-income countries: A systematic review and meta-analysis. Aids. 2012;26(16):2039-52.

25. Chaves RG, Lamounier JA. Uso de medicamentos durante a lactação. J Pediatr (Rio J). 2004 Nov;80(5):s189-98.

26. Feng X, Zhu W, Schurig-Briccio LA, Lindert S, Shoen C, Hitchings R, et al. Antiinfectives targeting enzymes and the proton motive force. Proc Natl Acad Sci. 2015 Dec;112(51):E7073-82.

27. Howard CR, Lawrence RA. Drugs and Breastfeeding. Clin Perinatol. 1999 Jun;26(2):447-78.

28. Svensson EM, Murray S, Karlsson MO, Dooley KE. Rifampicin and rifapentine significantly reduce concentrations of bedaquiline, a new anti-TB drug. J Antimicrob Chemother. 2014;70(4):1106-14.

29. Iscan M, Klaavuniemi T, Çoban T, KapucuoV Glu N, Pelkonen O, Raunio H. The expression of cytochrome P450 enzymes in human breast tumours and normal breast tissue. Breast Cancer Res Treat. 2001;70:47-54.

30. Hellmold H, Rylander T, Magnusson M, Reihner E, Warner M, Gustafsson J-A. Characterization of Cytochrome P450 Enzymes in Human Breast Tissue from Reduction Mammaplasties. J Clin Endocrinol Metab. 1998 Mar;83(3):886-95.

31. Huang Z, Fasco MJ, Figge HL, Keyomarsi K, Kaminsky LS. Expression of cytochromes P450 in human breast tissue and tumors. Drug Metab Dispos. 1996;24(8).

Table 1: Characteristics of pregnant women treated for rifampicin-resistant tuberculosis 


\begin{tabular}{|c|c|c|}
\hline Median (range) & Antepartum $(\mathrm{n}=13)$ & Postpartum $(\mathrm{n}=\mathrm{r}$ \\
\hline \multicolumn{3}{|l|}{ Baseline characteristics } \\
\hline Age (years) & $30(23-48)$ & $30(23-48)$ \\
\hline Height $(\mathrm{cm})$ & $160(140-176)$ & $162(152-163)$ \\
\hline HIV status (Pos/neg) & $(13 / 0)$ & $(6 / 0)$ \\
\hline TB type $\left(\mathrm{RR}^{\mathrm{a}} / \mathrm{MDR}^{\mathrm{b}} / \mathrm{Pre}-\mathrm{XDR}^{\mathrm{c}} / \mathrm{XDR} / \mathrm{missing}\right)$ & $(6 / 3 / 2 / 1 / 1)$ & $(2 / 2 / 0 / 1 / 1)$ \\
\hline Previous TB (yes/no/missing) & $(7 / 5 / 1)$ & $(2 / 3 / 1)$ \\
\hline CD4 (cells/mm3) & $311(44-1008)$ & $545(253-1008)$ \\
\hline $\mathrm{ART}^{\mathrm{d}}\left(\mathrm{NVP}^{\mathrm{e}} / \mathrm{LPV}^{\mathrm{f}} / \mathrm{DTG}^{\mathrm{g}}\right)$ & $10 / 1 / 2$ & $5 / 0 / 1$ \\
\hline Characteristics on the Pharmacokinetic day & Characteristics on the Pharmacokinetic day & Characteristic \\
\hline Weight $(\mathrm{Kg})$ & $61(55-104)$ & $67(52-84)$ \\
\hline Time since EFV ${ }^{\mathrm{h}}$ switch (Days) & $27(13-96)$ & $154(81-201)$ \\
\hline Gestational age/time after delivery (weeks) & $30(25-37)$ & $7(6.5-8)$ \\
\hline Inpatients/outpatients & $12 / 1$ & $0 / 6$ \\
\hline
\end{tabular}

$\mathrm{RR}^{\mathrm{a}}$ : rifampicin-resistant; $\mathrm{MDR}^{\mathrm{b}}$ : multidrug-resistant; $\mathrm{XDR}^{\mathrm{c}}$ : extremely drug-resistant; $\mathrm{ART}^{\mathrm{d}}$ : antiretroviral therapy; $\mathrm{NVP}^{\mathrm{e}}$ : nevirapine; LPV: lopinavir ${ }^{\mathrm{f}}$; DTG: Dolutegravirg $\mathrm{EFV}^{\mathrm{h}}$ : efavirenz

Table 2: Median (IQR) bedaquiline and metabolite (M2) concentrations per time point

\begin{tabular}{|c|c|c|c|c|c|c|c|c|}
\hline $\begin{array}{l}\text { Time } \\
\text { point }\end{array}$ & $\begin{array}{l}\text { Bedaquiline } \\
(\mathrm{n}=13)\end{array}$ & $\begin{array}{l}\text { Bedaquiline } \\
(\mathrm{n}=13)\end{array}$ & $\begin{array}{l}\text { Bedaquiline } \\
(\mathrm{n}=13)\end{array}$ & $\begin{array}{l}\text { Bedaquiline } \\
(\mathrm{n}=13)\end{array}$ & $\begin{array}{l}\text { Metabolite, } \\
\text { M2 } \\
(\mathrm{n}=6)\end{array}$ & $\begin{array}{l}\text { Metabolite, } \\
\text { M2 } \\
(\mathrm{n}=6)\end{array}$ & $\begin{array}{l}\text { Metabolite, } \\
\text { M2 } \\
(\mathrm{n}=6)\end{array}$ & $\begin{array}{l}\text { Metabolite, } \\
\text { M2 } \\
(\mathrm{n}=6)\end{array}$ \\
\hline & Antepartum & Antepartum & Postpartum & Postpartum & Antepartum & Antepartum & Antepartum & Postpartum \\
\hline Time & No. & $\begin{array}{l}\text { Concentratio } \\
(\mathrm{mg} / \mathrm{L})\end{array}$ & nNo. & $\begin{array}{l}\text { Concentratio } \\
(\mathrm{mg} / \mathrm{L})\end{array}$ & $\begin{array}{l}\text { (mg/L) } \\
(\mathrm{mg} / \mathrm{L} \text { tratio }\end{array}$ & no. & $\begin{array}{l}\text { Concentratio } \\
(\mathrm{mg} / \mathrm{L})\end{array}$ & no. \\
\hline \multirow[t]{3}{*}{ Predose } & \multirow[t]{3}{*}{6} & $\begin{array}{l}0.419 \\
(0.184\end{array}$ & 2 & $\begin{array}{l}0.186 \\
(0.161\end{array}$ & $\begin{array}{l}0.186 \\
(0.161\end{array}$ & \multirow[t]{3}{*}{6} & $\begin{array}{l}0.183 \\
(0.0922\end{array}$ & \multirow[t]{3}{*}{2} \\
\hline & & - & & - & - & & -0.218 & \\
\hline & & $0.711)$ & & $0.212)$ & $0.212)$ & & & \\
\hline \multirow[t]{3}{*}{$2 \mathrm{hrs}$} & \multirow[t]{3}{*}{6} & $\begin{array}{l}0.621 \\
(0.288\end{array}$ & 2 & $\begin{array}{l}0.237 \\
(0.221\end{array}$ & $\begin{array}{l}0.237 \\
(0.221\end{array}$ & 6 & $\begin{array}{l}0.160 \\
(0.115\end{array}$ & \multirow[t]{3}{*}{2} \\
\hline & & $-1.12)$ & & - & - & & - & \\
\hline & & & & $0.252)$ & $0.252)$ & & $0.231)$ & \\
\hline \multirow[t]{2}{*}{$4 \mathrm{hrs}$} & \multirow[t]{2}{*}{5} & $\begin{array}{l}1.05 \\
(0.863\end{array}$ & 1 & 1.06 & 1.06 & 5 & $\begin{array}{l}0.144 \\
(0.124\end{array}$ & \multirow[t]{2}{*}{1} \\
\hline & & $-1.32)$ & & & & & $\begin{array}{l}- \\
0.204)\end{array}$ & \\
\hline \multirow[t]{2}{*}{$6 \mathrm{hrs}$} & \multirow[t]{2}{*}{5} & $\begin{array}{l}1.69 \\
(1.59- \\
2.24)\end{array}$ & 1 & 1.14 & 1.14 & 5 & $\begin{array}{l}0.181 \\
(0.123 \\
-\end{array}$ & \multirow[t]{2}{*}{1} \\
\hline & & & & & & & $0.206)$ & \\
\hline $24 \mathrm{hrs}$ & 7 & $\begin{array}{l}0.308 \\
(0.287- \\
0.458)\end{array}$ & 4 & $\begin{array}{l}0.3085 \\
(0.298- \\
0.312)\end{array}$ & $\begin{array}{l}0.3085 \\
(0.298- \\
0.312)\end{array}$ & 7 & $\begin{array}{l}0.168 \\
(0.148- \\
0.458)\end{array}$ & 4 \\
\hline $26 \mathrm{hrs}$ & 7 & $\begin{array}{l}0.250 \\
(0.245- \\
0.443)\end{array}$ & 4 & $\begin{array}{l}0.281 \\
(0.269- \\
0.286)\end{array}$ & $\begin{array}{l}0.281 \\
(0.269- \\
0.286)\end{array}$ & 7 & $\begin{array}{l}0.142 \\
(0.132- \\
0.241)\end{array}$ & 4 \\
\hline
\end{tabular}




\begin{tabular}{|c|c|c|c|c|c|c|c|c|}
\hline $\begin{array}{l}\text { Time } \\
\text { point }\end{array}$ & $\begin{array}{l}\text { Bedaquiline } \\
(\mathrm{n}=13)\end{array}$ & $\begin{array}{l}\text { Bedaquiline } \\
(\mathrm{n}=13)\end{array}$ & $\begin{array}{l}\text { Bedaquiline } \\
(\mathrm{n}=13)\end{array}$ & $\begin{array}{l}\text { Bedaquiline } \\
(\mathrm{n}=13)\end{array}$ & $\begin{array}{l}\text { Metabolite, } \\
\text { M2 } \\
(\mathrm{n}=6)\end{array}$ & $\begin{array}{l}\text { Metabolite, } \\
\text { M2 } \\
(\mathrm{n}=6)\end{array}$ & $\begin{array}{l}\text { Metabolite, } \\
\text { M2 } \\
(\mathrm{n}=6)\end{array}$ & $\begin{array}{l}\text { Metabolite, } \\
\text { M2 } \\
(\mathrm{n}=6)\end{array}$ \\
\hline $28 \mathrm{hrs}$ & 7 & $\begin{array}{l}0.228 \\
(0.225- \\
0.391)\end{array}$ & 4 & $\begin{array}{l}0.275 \\
(0.269- \\
0.283)\end{array}$ & $\begin{array}{l}0.275 \\
(0.269- \\
0.283)\end{array}$ & 7 & $\begin{array}{l}0.145 \\
(0.138- \\
0.226)\end{array}$ & 4 \\
\hline $30 \mathrm{hrs}$ & 7 & $\begin{array}{l}0.205 \\
(0.182- \\
0.329)\end{array}$ & 3 & $\begin{array}{l}0.284 \\
(0.259- \\
0.292)\end{array}$ & $\begin{array}{l}0.284 \\
(0.259- \\
0.292)\end{array}$ & 7 & $\begin{array}{l}0.133 \\
(0.118 \\
- \\
0.205)\end{array}$ & 3 \\
\hline
\end{tabular}

No.: Number of participants at each timepoint

Figure 1

Visual predictive check (VPC) of the bedaquiline and M2, the top panels represent the parent, and the bottom panels represent the metabolite bedaquiline concentrations. The first column displays antepartum concentrations, while the last and middle columns shows postpartum concentration and antepartum concentration in participants co-administered lopinavir/ritonavir, respectively. Due to the small sample size in each panel, we plotted the $50^{\text {th }}$ percentiles of the observations (red line) - the shaded areas represent the 95\% model-predicted confidence intervals, and the black line is the median of the model predicted confidence interval.

Figure 2:

Pharmacokinetic profiles of bedaquiline concentrations stratified by participant ID. The red dots and purple crosses represent maternal plasma concentrations ante- and postpartum, respectively. The green triangles represent human milk; the blue squares represent infant plasma concentrations.

Figure 3:

Pharmacokinetic profiles of M2 concentrations stratified by participant ID. The red dots and purple crosses represent maternal plasma concentrations ante- and postpartum, respectively. The green triangles represent human milk; the blue squares represent infant plasma concentrations.

\section{Hosted file}

Figure1.docx available at https://authorea.com/users/446884/articles/546012-bedaquilineexposure-in-pregnancy-and-breastfeeding-in-women-with-rifampicin-resistant-tuberculosis

\section{Hosted file}

Figure2.docx available at https://authorea.com/users/446884/articles/546012-bedaquilineexposure-in-pregnancy-and-breastfeeding-in-women-with-rifampicin-resistant-tuberculosis

\section{Hosted file}

Figure3.docx available at https://authorea.com/users/446884/articles/546012-bedaquilineexposure-in-pregnancy-and-breastfeeding-in-women-with-rifampicin-resistant-tuberculosis 